\title{
Immunoadjuvant QS-DG
}

National Cancer Institute

\section{Source}

National Cancer Institute. Immunoadjuvant QS-DG. NCI Thesaurus. Code C74044.

A synthetic saponin, chemically identical to the natural saponin QS-21, with

immunoadjuvant activity. When co-administered with vaccine antigens, immunoadjuvant QS-DG may increase total antitumoral vaccine-specific antibody responses and cytotoxic T-lymphocyte (CTL) responses. 